\title{
Review
}

\section{Challenge and promise: roles for clusterin in pathogenesis, progression and therapy of cancer}

\author{
B Shannan ${ }^{1}$, M Seifert ${ }^{1}, \mathrm{~K}$ Leskov $^{2}$, J Willis ${ }^{3}$, D Boothman ${ }^{4}$, \\ W Tilgen ${ }^{1}$ and J Reichrath ${ }^{\star, 1}$ \\ 1 Department of Dermatology, The Saarland University Hospital, Homburg \\ 66421, Germany \\ 2 Department of Radiation Oncology, Case Western Reserve University, \\ Cleveland, OH, USA \\ ${ }^{3}$ Department of Pathology, Case Western Reserve University, Cleveland, $\mathrm{OH}$, \\ USA \\ ${ }^{4}$ Laboratory of Molecular Stress Responses, Department of Oncology, \\ University of Texas Southwestern Medical Centre at Dallas, Dallas, TX, USA \\ * Corresponding author: J Reichrath, Department of Dermatology, The Saarland \\ University Hospital, Homburg 66421, Germany. Tel: + 49-6841-1623802; \\ Fax: + 49-6841-1623845; E-mail: hajrei@med-rz.uni-saarland.de
}

Received 01.6.05; revised 16.8.05; accepted 18.8.05; published online 23.9.05 Edited by $\mathrm{J}$ Cleveland

\begin{abstract}
Clusterin (CLU) has been implicated in various cell functions involved in carcinogenesis and tumour progression. There are two known CLU protein isoforms generated in human cells. A nuclear form of CLU protein ( $\mathrm{nCLU}$ ) is proapoptotic, and a secretory form (sCLU) is prosurvival. CLU expression has been associated with tumorigenesis of various malignancies, including tumours of prostate, colon, and breast. Furthermore, CLU expression is modulated by many factors that are believed to regulate tumour growth and/or apoptosis, including 1,25-dihydroxyvitamin $D_{3}$, transforming growth factor beta-1, ultraviolet radiation, and IR. SCLU upregulation appears to be a general molecular stress response. Presently, preliminary results indicate that therapeutic modalities targeting CLU may be effective in cancer treatment. However, such strategies should make sure that $\mathrm{nCLU}$ is not eliminated or reduced. This review summarizes our present understanding of the importance of CLU in various physiological functions including tumour growth, and discusses its relevance to future cancer therapy.

Cell Death and Differentiation (2006) 13, 12-19.

doi:10.1038/sj.cdd.4401779; published online 23 September 2005
\end{abstract}

Keywords: clusterin; sCLU; nCLU; apoptosis; human tumours; DNA repair

Abbreviations: CLU, clusterin; SCLU, secretory clusterin; $\mathrm{nCLU}$, nuclear clusterin; pnCLU, precursor nuclear clusterin; NES, nuclear export sequence; NLS, nuclear localization signal; IR, ionizing radiation; siRNA, short interfering RNA; DNA-PK, DNA-dependent protein kinase; MMP, matrix metalloproteinase; DSB, double-strand break; $1,25(\mathrm{OH})_{2} \mathrm{D}_{3}, 1,25$-dihydroxyvitamin $\mathrm{D}_{3}$; TGF- $\beta 1$, transforming growth factor beta- 1 ; SBEs, Smad- binding elements; ASO, antisense oligonucleotides; Ad5CMVp53, adenoviral-mediated p53 gene transfer

\section{Introduction}

Clusterin (CLU), also known as apolipoprotein J, testosterone-repressed prostate message-2, or sulphated glycoprotein-2, is a secretory heterodimeric disulphide-linked glycoprotein (449 amino acids) that is expressed in virtually all tissues, and found in all human fluids. ${ }^{1-4}$ It is involved in numerous physiological processes important for carcinogenesis and tumour growth, including apoptotic cell death, cell cycle regulation, DNA repair, cell adhesion, tissue remodelling, lipid transportation, membrane recycling, and immune system regulation. ${ }^{1-3}$ Increased CLU mRNA and protein levels have been consistently detected in various tissues undergoing stress, including heart, brain, liver, kidney, breast, and retinal tissues both in vivo and in vitro. Early studies seemed to establish that CLU gene expression was a marker of apoptotic cell loss. ${ }^{5}$ Importantly, CLU upregulation has been reported in various human malignancies, including bladder, ${ }^{6}$ kidney, ${ }^{7}$ prostate, ${ }^{8-11}$ colon, ${ }^{3}$ breast, ${ }^{12,13}$ and lung ${ }^{14}$ tumours.

\section{Two CLU Isoforms with Distinct Biologic Activities}

Recent observations indicated an association of CLU expression with contradictory functions, either cell survival, tumour progression, treatment resistance in vivo, or apoptosis. ${ }^{2,4}$ These early apparently ambiguous functions appear to be attributed to the existence of two different but related CLU protein isoforms, a glycosylated as well as an apparently nonglycosylated form that are produced in the cell by alternative splicing. These two forms of the CLU protein can be immunologically distinguished. ${ }^{13}$

The secreted form of the CLU protein (sCLU) is a glycosylated protein of $76-80 \mathrm{kDa}$. Depending on the degree of glycosylation, it appears usually as two different protein bands by Western blot analyses: one full-length uncleaved $60 \mathrm{kDa}$ protein as well as another $\sim 40 \mathrm{kDa} \alpha$ and $\beta$ protein smear as observed by SDS-PAGE. ${ }^{13}$ sCLU expression is initiated by translation from the full-length CLU mRNA, wherein the first AUG codon is translated into the $49 \mathrm{kDa}$ sCLU precursor protein (Figure 1a). ${ }^{2,13}$ This protein is directed to the endoplasmic reticulum by a leader signalling sequence. As the protein is transported towards the Golgi, this form is cleaved at the $\alpha$ and $\beta$ site and heavily glycosylated. The result is a secreted protein of $\sim 80 \mathrm{kDa}$, which consists of $\alpha$ and $\beta$ peptides, linked by five disulphide bonds. This form appears as a smear at $\sim 40 \mathrm{kDa}$ smear on SDS-PAGE immunoblots (Figure 1). 
In contrast, nuclear clusterin (nCLU) is an initially synthesized $49 \mathrm{kDa}$ protein, referred to as a precursor to the nuclear form of the protein (i.e. pnCLU). In human cells, the nCLU protein was shown to be synthesized from a second in-frame AUG codon from an alternatively spliced nCLU mRNA, which was the result of the alternative splicing, which eliminated Exon II (which would otherwise encode for the sCLU start AUG and signalling leader peptide). It is currently unclear as to how nCLU is expressed in rodent cells, which apparently do not have conserved Exon I/II alternative splice sites that are present in all human cells. Once made, the $\sim 49 \mathrm{kDa}$ pnCLU protein does not undergo $\alpha / \beta$ cleavage, nor does this protein appear to be extensively glycosylated. pnCLU appears to be localized to the cytoplasm of normal human and rodent cells (Figure 1). ${ }^{15}$

In response to cell damage, the pnCLU protein is posttranslationally modified and a mature $\sim 55 \mathrm{kDa}$ proapoptotic protein form (nCLU) is generated that induces apoptosis. ${ }^{13}$ pnCLU is induced and translocated from the cytoplasm to the nucleus following certain cytotoxic events, including ionizing radiation (IR). ${ }^{13}$ Although its structure/function relationship is not completely understood, pnCLU appears to contain at least two nuclear localization signals that target the nCLU protein to the nucleus. Previous data demonstrated that overexpression of $\mathrm{nCLU}$ protein with both a C-terminal coiled-coil domain and a functional nuclear localization signal (NLS) only caused cell death. ${ }^{13}$ Some experiments have shown a mechanism by which the inert pnCLU could be activated to an nCLU 'death' protein. Recent data indicate that regulation of $\mathrm{nCLU}$ into the nuclei of irradiated cells occurs through both nuclear localization as well as nuclear export sequences (NES) (Leskov et al., unpublished data). These data suggest a very dynamic process in human cells wherein the conversion of pnCLU to $\mathrm{nCLU}$, as well as the continual export of nCLU protein regulates the overall signal for cell death in the cell. Further, studies to manipulate specifically mature nCLU levels inside stressed tumour compared to normal cells are underway in our laboratories. However, mutation in the NLS in the C-terminal appears to abrogate the majority of nCLU function. ${ }^{13}$

\section{CLU, Apoptosis, and Cell Cycle Regulation}

Although it has been suggested that CLU upregulation represents a marker of apoptotic response, the precise relationship between CLU gene expression and programmed cell death has not been elucidated clearly. ${ }^{16}$ Elucidation of the function of CLU in cells after stress is clearly complicated by the recognition of two different forms of the protein which apparently have two separate functions in the cell. A number of studies ${ }^{1,5,13,16,17}$ have demonstrated that overexpression of the full-length CLU mRNA that would produce, depending on where the translation would start from and in this case, $\mathrm{nCLU}$, acts as a prodeath signal, inhibiting cell growth and survival. However, other studies ${ }^{2,6,8,10,17-19}$ have shown that CLU expression may exert cytoprotective properties. We favour the recent proposal that tumour cell survival is connected with overexpression of SCLU and loss of nCLU. ${ }^{3}$
This theory has been supported by recent data which suggest that cells must suppress sCLU to stimulate cell death. One proposed mechanism for this death process is through the activation of the p53 tumour suppressor gene. ${ }^{17}$ This would apparently explain how progression towards high-grade and metastatic carcinoma leads to a shift from nCLU to sCLU expression, and that the role of CLU for tumour growth may be related to a pattern shift in its isoform production. ${ }^{3}$

It is well known that apoptotic pathways are regulated via an orchestrated interplay of multiple activators (e.g. tumour necrosis factor, Fas ligand, Bax, and p53) and suppressors (e.g. various growth factors and $\mathrm{Bcl}-2$ ) ${ }^{20}$ In this context, it is interesting that $\mathrm{p53}$, an activator of the apoptotic cascade, can suppress basal as well as IR-induced SCLU expression in both MCF-7 (breast cancer) and HCT116 (colon cancer) cells by repressing CLU promoter activity and transcription. ${ }^{17}$ Recent clinical results by our group appear to support this theory in vivo (Willis and Boothman et al., unpublished data), in which loss of functional p53 appears to result in lost nCLU function. We are currently working on the mechanism through which sCLU expression is downregulated by p53 and nCLU functionally lost if p53 is mutated or cells lose expression of this tumour suppressor.

CLU expression was reported to be directly regulated by $B$ $M Y B$, a transcription factor involved in the regulation of cell survival, proliferation, and differentiation. ${ }^{21}$ Another transcription factor that was reported to be regulated by $\mathrm{CLU}$ is $\mathrm{NF}-\kappa \mathrm{B}$. Modulation of NF- $\kappa \mathrm{B}$ activity is believed of importance for cell survival, cell motility, proliferation, and transformation. Its activity is regulated through interaction with inhibitory molecules ( $\left.I_{\kappa} \mathrm{Bs}\right)$. CLU expression could be required to suppress excessive NF- $\kappa$ B activation, which determines inflammation and cardiomyocyte apoptosis in autoimmune myocarditis in the rat. Loss of CLU expression in cells that depend on NF- $\kappa \mathrm{B}$ activity for proliferation or chemoresistance could lead to tumour progression. For example, acutely expressed CLU can significantly reduce the in vitro invasive properties of neuroblastoma cells. However, it is not clear yet how CLU regulates $\mid \kappa$ Bs protein turnover. ${ }^{22}$

To what extent the expression of CLU is cell cycle dependent is still a matter of debate. It was shown that in human dermal fibroblasts CLU acts as a cell cycle-related gene, controlled by serum starvation, and that CLU protein accumulation occurred in the $G_{0}$ phase in human dermal fibroblasts. ${ }^{23}$ CLU overexpression in immortalized human prostate epithelial cells resulted in an increased accumulation of cells at the $G_{0} / G_{1}$ phases of the cell cycle, accompanied by slow down of cell cycle progression and a reduction of DNA synthesis. ${ }^{11}$ CLU overexpression also resulted in DNA damage accumulation in LNCaP prostate cells, and helped accelerate prostate cancer development. This is thought to have occurred, at least in part, through the inhibition of membrane damage induced by $\mathrm{H}_{2} \mathrm{O}_{2}{ }^{8}$. High levels of nCLU were reported to cause $\mathrm{G}_{1}$ cell cycle arrest in distinct cell types. ${ }^{16}$ The overall function of CLU expression in cell cycle regulation remains unclear. Use of short interfering RNAs (siRNAs) specific to each form of the CLU proteins should allow future studies to delineate cause-effect relationships and reveal whether SCLU or nCLU directly affect cell cycle regulation with or without cell stress. 


\section{CLU and DNA Repair}

In addition to the function of CLU in mediating apoptosis, ${ }^{1,5,13,16,17}$ increasing evidence supports an important function for CLU in DNA repair. ${ }^{23}$ Interestingly, CLU has been identified as the first stress-inducible protein that is associated with the DNA-dependent protein kinase (DNA-PK) double-strand break (DSB) repair complex. ${ }^{16} \mathrm{nCLU}$, but not sCLU, protein binds to Ku70, forming a trimeric protein complex with Ku80; Ku70 and Ku80 are two components of the DNA-PK complex, involved in nonhomologous DNA DSB repair. ${ }^{16,24}$ DNA-PK is a nuclear serine/threonine kinase that requires DNA ends for catalytic activity. It is selectively activated by binding to the ends of linear doublestrand DNA. The exact mechanism by which DNA-PK takes part in the physical resealing of a DSB is not fully understood. ${ }^{16,25}$ Data showing that overexpression of CLU reduces the binding activity of Ku70/Ku80 to DNA ends in whole-cell extracts suggests that nCLU may directly affect DSB repair. However, the importance of $\mathrm{nCLU}$ in DNA repair versus the induction of apoptosis remains to be elucidated. ${ }^{16}$ Recent data by our group indicate that Ku70 plays an important dual role in binding Bax and protecting cells from Bax-mediated cell death responses. ${ }^{24,26}$ We are currently testing the hypothesis that nCLU acts to release Bax from the cytoprotective Ku70-Bax complex, as well as prevent nonhomologous end joining through the inhibition of Ku70-Ku80 end binding activity. ${ }^{26}$

\section{CLU, Cell Matrix Formation, Cell Membrane Remodelling, and Morphological Modulation}

It has been demonstrated that CLU may act as a negative regulator of MT6-matrix metalloproteinase (MMP) in vivo, a component of the membrane-type MMPs subgroup in the MMP family. MT6-MMP is expressed in neutrophils and in some brain tumours, and seems to play a vital role in neutrophil function. ${ }^{27}$ However, the MMPs are responsible for the degradation of most components of the extracellular matrix, and the MT6-MMP specifically produced by neutrophils are believed to be important for invasion and migration of cells to inflammatory sites, and/or destruction of host tissue. ${ }^{27}$ The importance of CLU expression for tissue remodelling and apoptosis has not only been demonstrated in malignancies but also in other models of cell growth and tissue remodelling, including in hair growth. ${ }^{28}$ The hair follicle goes through cycles of growth, regeneration, and rest that involve tissue remodelling and apoptosis. Expression of SCLU during the growth phase of the hair cycle suggests that this protein may have a role in the maintenance of the layered structure of the hair follicle. ${ }^{28}$

A study using smooth muscle cells showed that morphological modulation associated with a change from a monolayer to a nodular cell culture resulted in increased levels of CLU mRNA and protein. $^{29}$ Moreover, endogenous sCLU was found to support rapid nodular formation and is proposed to play a functional role in cell matrix. ${ }^{29}$
These findings demonstrate an important role for CLU for cell matrix formation, cell membrane remodelling, and morphological tissue modulation under various physiological and pathological conditions.

\section{Regulation of CLU Expression in Cancer Cells}

A broad variety of different compounds involved in the modulation of tumour growth or in cancer therapy can regulate CLU expression in cancer cells. 1,25-Dihydroxyvitamin $D_{3}$ $\left(1,25(\mathrm{OH})_{2} \mathrm{D}_{3}\right)$ or its analogues inhibit proliferation and induce apoptosis in a broad variety of cell lines, including cancer cells. ${ }^{13,20,30-32}$ Increased expression of both sCLU and a prostatic atrophy marker were observed after treatment of benign prostate hyperplastic cells with the $1,25(\mathrm{OH})_{2} \mathrm{D}_{3}$ analogue, BXL-628. ${ }^{33} \mathrm{BXL}-628$ induced inhibition of prostate cell proliferation in vivo and in vitro. $\mathrm{SCLU}$ overexpression in LNCaP cells (some LNCaP clones express very low levels of sCLU) may help accelerate prostate cancer development through the inhibition of membrane damage induced by $\mathrm{H}_{2} \mathrm{O}_{2} .{ }^{8}$ Recently, we have shown a time-dependent upregulation of CLU gene expression in MeWo, and SK-MEL-28 melanoma cells after treatment with $1,25(\mathrm{OH})_{2} \mathrm{D}_{3}$ at various time points (Shannan et al., under review, Journal of Cellular Biochemistry).

Other factors that have been shown to affect the expression of CLU in tumour cells include various growth factors that mediate proliferation and differentiation signals that are important for tumour cell growth. CLU mRNA was induced and activated in PC12 prostate cancer cells by nerve growth factor and epidermal growth factor. ${ }^{34}$ Transforming growth factor beta (TGF- $\beta$ ) induces CLU gene expression in a variety of cell types. Transforming growth factor beta-1 (TGF- $\beta 1$ ) was shown to enhance sCLU protein and mRNA expression in thyroid epithelial cells, and it was proposed that upregulation of CLU expression may be a marker of TGF- $\beta 1$-mediated thyrocyte dedifferentiation. ${ }^{34}$ In rodent cells, CLU gene expression was proposed to occur via a consensus AP-1-binding site located in the promoter region of this gene. ${ }^{15}$ Furthermore, TGF- $\beta 1$-induced CLU expression in CCL64 mink lung cancer cells was repressed by overexpression of the c-Fos proto-oncogene, and it was speculated that TGF- $\beta 1$ could exert effects on $\mathrm{c}$-Fos protein synthesis and/or stability, resulting in the repression of c-Fos, and thus increased CLU gene expression. ${ }^{15}$ More recent data from our group has shed additional light on TGF- $\beta 1$-induced expression, differentiating CLU promoter regulation from other agents (e.g. IR). ${ }^{35}$ These data indicate that while CLU promoter activity is regulated through Egr-1 and Egr-1 consensus sites in the CLU promoter after IR, TGF- $\beta 1$ regulates CLU expression by activation of Smad 3 and 4 binding to three highly conserved Smad-binding elements (SBEs). ${ }^{36}$ Although both IR and TGF- $\beta 1$ upregulate $\mathrm{SCLU}$ expression in human and rodent cells, the mechanism by which CLU expression is induced appear to vary with the cytotoxic or cytostatic agent. 


\section{Expression of CLU in Malignant Tumours and its Potential Role as a Prognostic Marker}

Changes in SCLU expression have been documented in a broad variety of different malignancies including in human prostate, skin, pancreatic, breast, lung, and colon tumours, as well as in oesophageal squamous cell carcinoma, and neuroblastoma. ${ }^{2}$ It is now accepted that the primary function of SCLU in distinct genetic backgrounds of cancer cells is antiapoptotic. $^{2}$ However, whether increased expression of antiapoptotic sCLU is a common feature of tumorigenesis, thereby protecting cancer cells against apoptotic stimuli that might cause cell death, is still a matter of debate. Moreover, the question whether antiapoptotic sCLU is the only form of CLU expressed in cancer, or whether proapoptotic forms of nCLU are downregulated in distinct tumour entities (as discussed above), has not been definitely answered to date.

Recent data indicate that progression towards high-grade and metastatic carcinoma leads to elevated SCLU levels and altered intracellular distribution of nCLU. Thus, the function of CLU in tumours may be related to a pattern shift in its isoform

a

Coiled-Coil

NLS

$\mathrm{NH}_{3}-$

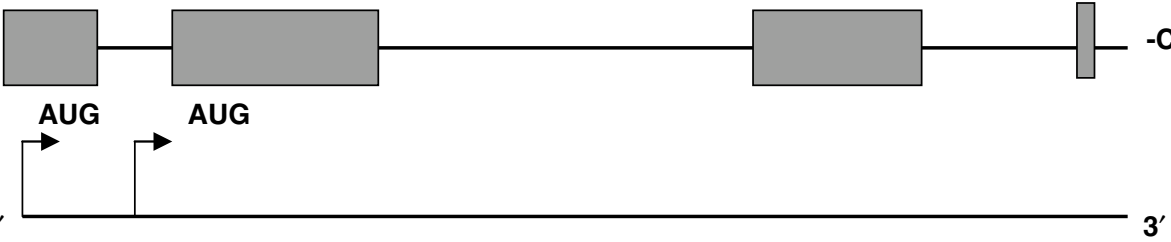

$-\mathrm{COOH}$

CLU Protein

CLU mRNA

sCLU precursor $49 \mathrm{kDa}$

pnCLU 48 kDa

b

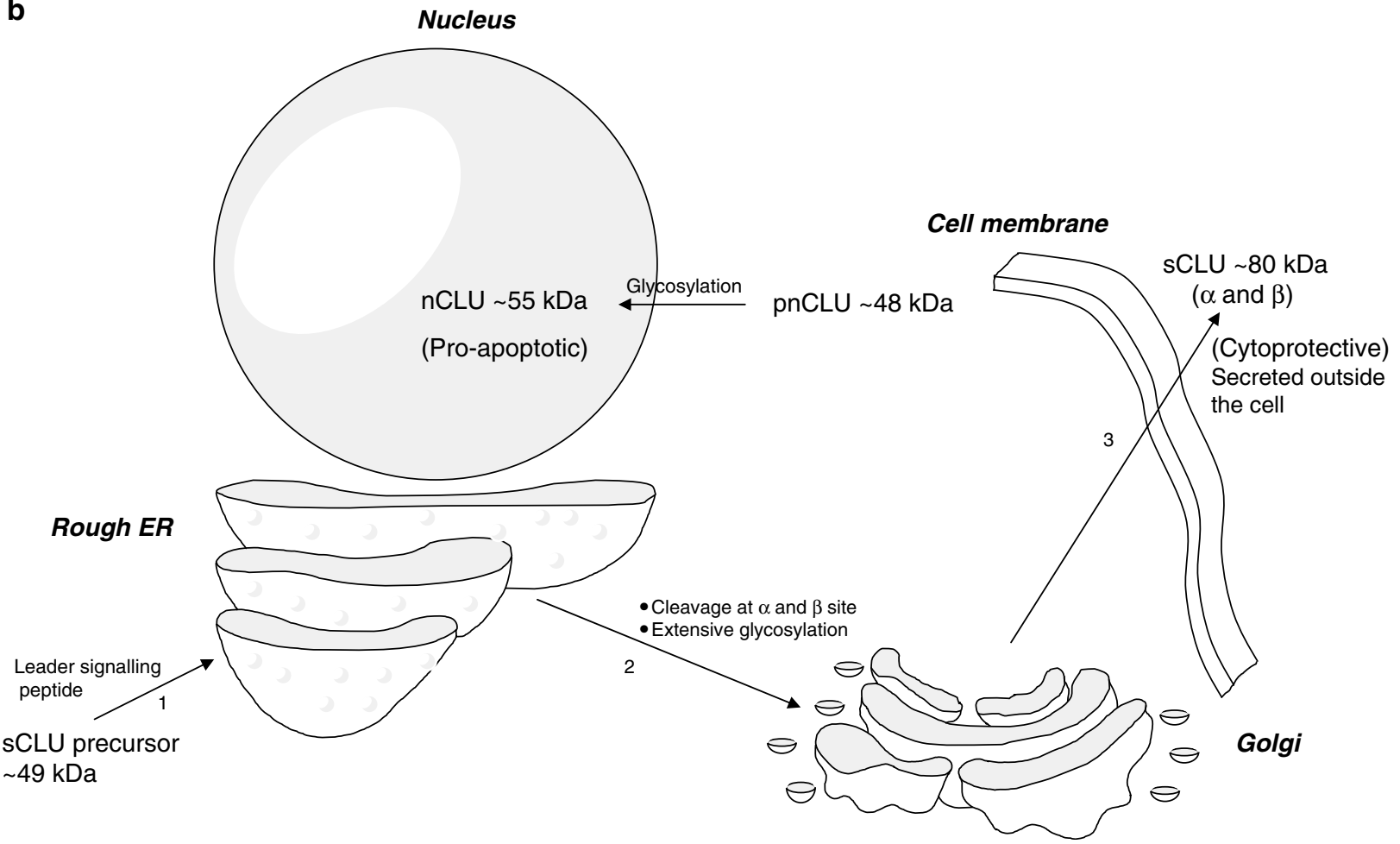

Figure 1 Generation of sCLU and $n C L U$. (a) sCLU precursor is translated from the first AUG codon of the full-length CLU mRNA, while $n C L U$ precursor (pnCLU) is obtained by alternative splicing from the second in-frame AUG codon of the full-length CLU mRNA. LP: leader peptide; NLS: nuclear localization signal. (b) sCLU precursor is transported into the rough ER by leader signalling peptide (1), and then undergoes cleavage and extensive glycosylation while being transported to the Golgi apparatus (2). The result is a secreted protein of $\sim 80 \mathrm{kDa}$ with five disulphide bonds between the $\alpha$-and $\beta$-subunit that is secreted outside the cell (3). pnCLU does not undergo any cleavage nor extensive glycosylation, and resides in the cytoplasm of unstressed cells. It becomes the mature form $(\sim 55 \mathrm{kDa})$ once it is transported to the nucleus 
production. ${ }^{3}$ As discussed above, overexpression of sCLU was shown in the majority of tumours investigated including prostate cancer, ${ }^{8,18,19,37}$ breast carcinoma, ${ }^{12,38}$ lung, ${ }^{14}$ bladder, $^{6}$ and colon $^{3}$ cancers. In fact, CLU upregulation was closely associated with disease progression and recurrence in patients with bladder cancer. ${ }^{6}$ Full-length CLU mRNA and sCLU protein were both involved in intestinal tumours. ${ }^{39}$ In prostate cancer, sCLU expression was associated with the development of androgen resistance and it (SCLU) is thought to act as a protective agent against treatment-induced cell death. ${ }^{8,38}$ In fact, not many studies showed a decrease in sCLU in tumours like that of a proapoptotic. A few reports do, however, suggest decreased SCLU levels in specific cancers, including nonmelanoma skin cancer, ${ }^{40}$ oesophageal squamous cell carcinoma, ${ }^{41}$ neuroblastoma, ${ }^{22}$ prostate, ${ }^{11,37}$ and pancreatic cancer $^{42}$ (Table 1).

In one study, CLU expression was analysed during progression of prostate cancer. ${ }^{37}$ While expression levels of sCLU protein and full-length CLU mRNA were almost undetectable in prostate carcinoma cells, high expression levels of CLU protein and mRNA were found in stromal peritumoral fibroblasts. In different stages of tumour progression, sCLU accumulated in specific, well-defined areas of the stromal compartment of the tumours. In advanced prostate cancer, staining of the remnants of the extracellular matrix suggested a role for sCLU in the process of dismantling the stromal organization caused by prostate cancer. ${ }^{37} \mathrm{CLU}$ expression was induced in pancreatic cancer and its downregulation may be associated with disease progression. ${ }^{42}$ It is not clear, however, in these studies if sCLU versus nCLU expression levels were clearly separable. There is a need for improved methods to detect nCLU in cases like this. Thus, although the majority of studies indicate that SCLU expression is upregulated along with the progression of distinct malignancies, its role as a prognostic factor is still questionable and more research examining expression levels of SCLU versus $\mathrm{nCLU}$ in these cancers is needed. $3,6,8,9,18,39$

In contrast, sCLU levels have been implicated as having prognostic value in specific cancers. For example, sCLU was reported to be a prognostic indicator in tumorigenesis and progression of human breast carcinoma. ${ }^{38}$ Furthermore, sCLU has been proposed to be a potentially new prognostic and predictive marker for colon carcinoma aggressiveness, since overexpression of sCLU is reported or observed in

Table 1 Summary of CLU expression in various tumours

\begin{tabular}{|c|c|}
\hline Tumour & $\begin{array}{l}\text { CLU expression (as observed } \\
\text { by previous studies) }\end{array}$ \\
\hline Prostate cancer $5,10,18,19$ & $\uparrow *$ mRNA and protein \\
\hline Breast carcinoma $^{12,13,35}$ & $\uparrow \mathrm{mRNA}$ \\
\hline Lung cancer ${ }^{14}$ & $\uparrow \mathrm{mRNA}$ \\
\hline Colon cancer ${ }^{3,28}$ & $\uparrow$ mRNA and protein \\
\hline Skin cancer ${ }^{38}$ & $\downarrow * \star$ mRNA \\
\hline $\begin{array}{l}\text { Oesophageal squamous } \\
\text { cell carcinoma } 39\end{array}$ & $\downarrow$ Protein \\
\hline Neuroblastoma cancers ${ }^{40}$ & $\downarrow$ Protein \\
\hline Pancreatic cancer ${ }^{41}$ & $\downarrow$ mRNA \\
\hline Prostate cancer ${ }^{11,37}$ & $\downarrow$ mRNA \\
\hline
\end{tabular}

$\uparrow^{\star}$ : Increase in expression; $\downarrow^{\star \star}$ : decrease in expression highly aggressive tumours as well as metastatic nodules. ${ }^{3}$ In addition, in prostate LNCaP cells, CLU appeared to be associated with tumour progression and resistant to chemotherapy treatments in vivo. ${ }^{18}$ However, preliminary data show that the pattern of distribution of full-length CLU mRNA and sCLU protein expression in prostate tumours does not seem to be affected by treatment modalities. ${ }^{37}$

Therefore, as with overall basal levels of sCLU, the notion of whether changes in SCLU expression may be an indicator of treatment response is still to be elucidated. Recent preliminary data from our laboratory indicate a strong correlation between sCLU expression and resistance to therapy of advanced human breast cancer patients.

\section{CLU as a Diagnostic Marker in Malignancies}

The SCLU antigen has been characterized as a sensitive and stable histological marker for murine and human intestinal tumours. ${ }^{39}$ This property can make sCLU be considered as a potential biomarker for diagnosis of human colorectal cancer using immunohistochemical or molecular biology techniques. Furthermore, elevated production of SCLU antigen, if secreted from tumour cells, may be detected in body fluids, such as serum. Consequently, it has been speculated that markers derived from sCLU may be used as part of a set of indices for early detection of human colorectal cancer. ${ }^{39} \mathrm{sCLU}$ was also identified as a marker of anaplastic large-cell lymphoma ${ }^{43}$ and it has been recommended that SCLU should be added as a useful marker to antibody panels designed to distinguish systemic anaplastic large-cell lymphoma from classical Hodgkin's lymphoma. ${ }^{43}$ These findings show that an increasing body of evidence now indicates that the specific expression patterns of sCLU and its correlation with cellular events during tumorigenesis make it a promising diagnostic tool in anaplastic large-cell lymphoma, as well as other malignancies. Thus, sCLU is a potential contributor to the set of biomarkers for early detection of a variety of cancers, especially colon cancer.

\section{CLU as a Therapeutic Target in Malignancies}

Recent scientific findings demonstrate that drugs targeting sCLU expression, including CLU silencing using antisense oligonucleotides (ASO) or short interfering double-stranded RNA, may become promising tools for cancer therapy. ${ }^{4,51}$ It is now accepted that the primary function of the $67-80 \mathrm{kDa}$ sCLU protein form is cytoprotective and it is well known that resistance to cancer treatment is mediated, at least in part, by enhanced expression of cell survival proteins that help facilitate tumour progression. ${ }^{9}$ Thus, it has become increasingly clear that in most cells SCLU is a stress-associated cytoprotective protein that is upregulated by various apoptotic triggers. Furthermore, sCLU confers resistance by some unknown mechanism when overexpressed. ${ }^{9}$ The novel therapeutic strategy of silencing sCLU expression to overcome resistance to cancer therapy is of interest for the treatment of cancers that overexpress SCLU as in kidney, 
Table 2 Summary of CLU ASOs used in various tumours

\begin{tabular}{|c|c|c|c|c|}
\hline Tumour & Study type & ASO & $\begin{array}{l}\text { Additional } \\
\text { treatment }\end{array}$ & Outcome of treatment \\
\hline Breast $^{45}$ & Phase I trial & OGX-011 & $\begin{array}{l}\text { Trastuzumab and/or } \\
\text { Bcl-2 ASO }\end{array}$ & $\begin{array}{l}\text { Combined therapy: activation of apoptosis (not observed in } \\
\text { either agents alone) }\end{array}$ \\
\hline Prostate $^{46}$ & Phase I trial & OGX-011 & Chemotherapy & $\begin{array}{l}\text { Combined therapy: improved efficiency of chemotherapy, } \\
\text { radiation, and androgen withdrawal by enhancing the } \\
\text { apoptotic response (not observed in chemotherapy alone) }\end{array}$ \\
\hline$P C-3^{47}$ & $\begin{array}{l}\text { In vitro and } \\
\text { in vivo }\end{array}$ & CLU ASO & $\begin{array}{l}\text { Ad5CMV-p53 } \\
\text { (adenoviral- } \\
\text { mediated p53 gene } \\
\text { transfer) }\end{array}$ & $\begin{array}{l}\text { Combined treatment: inhibition of PC-3 tumour growth } \\
\text { compared to no growth-inhibitory effect using ASO alone, } \\
\text { reduction of the } \mathrm{IC}_{50} \text { of Ad5CMV-p53 by } 75 \%\end{array}$ \\
\hline Kidney (Caki-2) ${ }^{48}$ & $\begin{array}{l}\text { In vitro and } \\
\text { in vivo }\end{array}$ & CLU ASO & $\begin{array}{l}\text { Cytotoxic } \\
\text { chemotherapy } \\
\text { Paclitaxel }\end{array}$ & $\begin{array}{l}\text { Combined treatment: enhanced Caki-2 cells apoptosis } \\
\text { while ASO alone had no effect on cells' growth } \\
\text { Combined treatment: suppressed tumour growth by } 50 \% \\
\text { as compared to treatment with mismatch control } \\
\text { oligonucleotides plus paclitaxel }\end{array}$ \\
\hline Bladder (KoTCC-1) ${ }^{6}$ & $\begin{array}{l}\text { In vitro and } \\
\text { in vivo }\end{array}$ & CLU ASO & Cisplatin & $\begin{array}{l}\text { Combined treatment: delayed tumour progression, and } \\
\text { enhanced cisplatin-induced apoptosis compared to no } \\
\text { growth-inhibitory effect using ASO alone. Resistant tumour } \\
\text { phenotypes arise with the progression of the disease when } \\
\text { cisplatin is used alone }\end{array}$ \\
\hline Lung (A549) $)^{14}$ & $\begin{array}{l}\text { In vitro and } \\
\text { in vivo }\end{array}$ & CLU ASO & Paclitaxel & $\begin{array}{l}\text { Combined treatment: enhanced the effects of paclitaxel to } \\
\text { significantly delay the growth of the tumour compared to } \\
\text { mismatch control oligonucleotides }\end{array}$ \\
\hline
\end{tabular}

prostate ${ }^{44}$ colon, breast, and lung tumours ${ }^{14}$ (Table 2). Recent findings support the concept that silencing sCLU expression can enhance the cytotoxicity of various chemotherapeutic agents ${ }^{1,49}$ as well as IR. ${ }^{50}$ However, a potential disadvantage of ASO therapy is the rapid intracellular degeneration of oligonucleotides. This problem was overcome by replacing a nonbridge phosphoryl oxygen of DNA with a sulphate to create a phosphothiate backbone, thus creating a first generation of ASOs intended for cancer therapy. ${ }^{46}$ Considerable efforts have been made to improve efficacy of ASO-based cancer therapy. Modifications made to the ribose, the 2'-position, improve the pharmacokinetic characteristics of second-generation phosphothioate ASOs, for example, OGX-011. ${ }^{46}$ In advanved breast cancer, ${ }^{45}$ OGX011 targeting CLU enhanced the growth-inhibitory effect of Trastuzumab, an HER-2-targeted monoclonal antibody used in the clinical management of advanced breast cancer patients. Only the combination of OGX-011 and Tratuzumab lead to an activation of apoptosis that was not observed with either agents alone. It has been suggested that a reduction in CLU expression by ASO may enhance the sensitivity of the cytotoxic chemotherapy for advanced breast cancer. ${ }^{45}$ Phase I studies have suggested a combined therapy using ASOs against CLU and Bcl-2, along with Trastuzumab for advanced breast cancer patients. Phase II studies are still underway. ${ }^{45}$

Phase I trials in prostate cancer have shown that OGX-011 improved the efficacy of chemotherapy, radiation, and androgen withdrawal by inhibiting the expression of CLU and enhancing the apoptotic response. Phase II trails combining OGX-011 and chemotherapy are still being assessed. ${ }^{46}$ In other prostate cancer phase I trails, OGX011 was given prior to radical prostoctomy, while some 'welltolerated' phase II trials were established based on the biologic effectiveness of OGX-011. ${ }^{4}$ The use of CLU ASO on Caki-2 cells (human clear cell carcinoma of the kidney) inhibited CLU mRNA expression. ASO against CLU alone showed no effect on Caki-2 cells' growth. ${ }^{48}$ CLU ASO combined with cytotoxic chemotherapy enhanced Caki-2 cells apoptosis both in vivo and in vitro, while CLU ASO combined with paclitaxel suppressed Caki-2 tumour growth by $50 \%$ as compared to treatment with mismatch control oligonucleotides plus paclitaxel. ${ }^{48}$ Treatment of KoTCC-1 (human bladder cancer cells) in vivo and in vitro with CLU ASO enhanced the $\mathrm{IC}_{50}$ of cisplatin by more than $50 \%$ and enhanced cisplatin-induced apoptosis, while no effect was observed upon KoTCC-1 treatment with CLU ASO alone. ${ }^{6}$ Most bladder cancer cases initially respond to cisplatin-based combination chemotherapy, but resistant phenotypes develop with the progression of the disease. Thus, cisplatin (at tolerable doses) alongside CLU ASO delays tumour progression. ${ }^{6}$ Similar results have shown that nucleotide-based therapies targeting sCLU (sCLU silencing using ASO or siRNA) chemosensitize human lung adenocarcinoma cells both in vitro and in vivo. ${ }^{14}$ In these studies, the effect of SCLU ASO or siRNA treatment on SCLU expression and chemosensitivity to paclitaxel was examined in A549 lung adenocarcinoma cells in vitro, while in vivo administration of SCLU ASO (compared to mismatch control oligonucleotides) synergistically enhanced the effects of paclitaxel or gemcitibine to significantly delay growth of A549 tumours in immunocompromised mice. In vitro and in vivo studies demonstrated that combined treatment of CLU ASO and paclitaxel reduced the mean PC-3 tumour volumes more efficiently. ${ }^{51}$ Treatment of PC-3 cells with CLU ASO reduced the $\mathrm{IC}_{50}$ of apoptosisinducing agent Ad5CMV-p53 (adenoviral-mediated p53 gene transfer) by $75 \%$, although no growth-inhibitory effects were observed in PC-3 cells treated with CLU ASO alone. ${ }^{47}$ In vivo, combined treatment of CLU ASO and Ad5CMV-p53 inhibited the growth of PC-3 tumours. ${ }^{47}$ Moreover, it has been demonstrated that treatment of PC-3 prostate cancer cells with CLU ASO significantly enhanced IR-induced apoptosis and decreased cellular growth. ${ }^{18}$ Administration of CLU ASO 
in vivo before and after radiation significantly reduced $\mathrm{PC}-3$ tumour volume by $50 \%$ as compared to radiation alone, ${ }^{10,18}$ Table 2. It has been hypothesized that SCLU overexpression may influence the apoptotic response of prostate tumour cells to IR and that the upregulation of CLU after various apoptotic signals represents an adaptive cell survival mechanism, thereby affecting a potential target for downregulation to improve the results of radiation therapy for patients with prostate cancer. ${ }^{18}$ Thus, sCLU overexpression helps confer a chemoresistant phenotype through inhibition of apoptosis in human renal cell carcinoma cells. ${ }^{7}$ Analysis of the functional role of sCLU in chemotherapy-induced apoptosis suggests that lowering the expression of SCLU might increase the sensitivity of bladder cancer cells to chemotherapeutic agents. $^{52}$ Furthermore, sCLU was supported as a valid therapeutic target in strategies employing novel multimodality therapy for advanced prostate cancer, as SCLU is upregulated following androgen therapy, and this may represent an adaptive cell survival response following apoptotic signals like androgen withdrawal. ${ }^{44}$

Consequently, it has been hypothesized that SCLU gene silencing using siRNA or other techniques may ultimately develop into attractive antitumour therapeutics. ${ }^{2}$ It has recently been shown that SCLU knockdown in human cancer cells, using siRNA-mediated CLU gene silencing, induces significant reduction of cellular growth and higher rates of spontaneous endogenous apoptosis. ${ }^{2}$ sCLU provides cytoprotection for IR-exposed MCF-7 breast cancer cells that is not observed after siRNA to silence CLU gene. ${ }^{50}$ However, great care in reducing sCLU levels without affecting nCLU levels must be taken using siRNA targeted against a specific CLU isoform. siRNA knockdown sCLU levels enhances cytotoxic agent efficacy, whereas siRNA specific to sCLU enhances efficacy of cytotoxic agents, whereas siRNA against nCLU confers cytoprotection against a variety of chemotherapeutics.

\section{Future Outlook}

In conclusion, a large body of evidence now supports important functions of CLU expression in the pathogenesis and progression of cancer. Although recent findings are very promising, future studies will have to meet the challenge to clarify specifically the role(s) of different CLU isoforms as diagnostic or prognostic markers for cancer malignancies, and as potential targets for therapeutic modalities for effective cancer therapy.

\section{References}

1. Trougakos IP and Gonos ES (2002) Clusterin/apolipoprotein J in human ageing and cancer. Int. J. Biochem. Cell Biol. 34: 1430-1448

2. Trougakso IP, So A, Jansen B, Gleave ME and Gonos ES (2004) Silencing expression of the clusterin/apolipoprotein $\mathrm{J}$ gene in human cancer cells using small interfering RNA induces spontaneous apoptosis, reduced growth ability, and cell sensitization to genotoxic and oxidative stress. Cancer Res. 64: 18341842

3. Pucci S, Bonanno E, Pichiorri F, Angeloni C and Spagnoli LG (2004) Modulation of different clusterin isoforms in human colon tumorigenesis. Oncogene 23: 2298-2304
4. Gleave M and Miyake $\mathrm{H}$ (2005) Use of antisense oligonucleotides targeting the cytoprotective gene, clusterin, to enhance androgen- and chemo-sensitivity in prostate cancer. World J. Urol. 23: 38-46

5. Lakins J, Steffany A, Bennett L, Chen JH, Arnold JM, Morrissey C, Wong P, O'Sullivan J and Tenniswood M (1998) Clusterin biogenesis is altered during apoptosis in the regressing rat ventral prostate. J. Biol. Chem. 273: 2788727895

6. Miyake H, Hara I, Kamidono S and Gleave ME (2001) Synergistic chemosensitization and inhibition of tumor growth and metastasis by the antisense oligodeoxynucleotide targeting clusterin gene in a human bladder cancer model. Clin. Cancer Res. 7: 4245-4252

7. Hara I, Miyake H, Gleave ME and Kamidono S (2001) Introduction of clusterin gene into human renal cell carcinoma cells enhances their resistance to cytotoxic chemotherapy through inhibition of apoptosis both in vitro and in vivo. Jpn. J. Cancer Res. 92: 1220-1224

8. Miyake H, Hara I, Gleave ME and Eto H (2004) Protection of androgendependent human prostate cancer cells from oxidative stress-induced DNA damage by overexpression of clusterin and its modulation by androgen. Prostate 61: 318-323

9. Miyake H, Nelson C, Rennie PS and Gleave ME (2000) Testosteronerepressed prostate message-2 is an antiapoptotic gene involved in progression to androgen independence in prostate cancer. Cancer Res. 60: 170-176

10. Zellweger $\mathrm{T}$, Chi K, Miyake $\mathrm{H}$, Adomat $\mathrm{H}$, Kiyama S, Skov $\mathrm{K}$ and Gleave ME (2002) Enhanced radiosensitivity in prostate cancer by inhibition of the cell survival protein clusterin. Clin. Cancer Res. 8: 3276-3284

11. Bettuzzi S, Scorcioni F, Astancolle S, Davalli P, Scaltriti M and Corti A (2002) Clusterin (SGP-2) transient overexpression decreases proliferation rate of SV40-immortalized human prostate epithelial cells by slowing down cell cycle progression. Oncogene 21: 4328-4334

12. van Weelden K, Flanagan L, Binderup L, Tenniswood M and Welsh JE (1998) Apoptotic regression of MCF-7 xenografts in nude mice treated with the vitamin D3 analogue, EB1089. Endocrinology 139: 2102-2110

13. Leskov KS, Klokov DY, Li J, Kinsella TJ and Boothman DA (2003) Synthesis and functional analyses of nuclear clusterin, a cell death protein. J. Biol. Chem. 278: 11590-11600

14. July LV, Beraldi E, So A, Fazil L, Evans K, English JC and Gleave ME (2004) Nucleotide-based therapies targeting clusterin chemosensitized human lung adenocarcinoma cells both in vitro and in vivo. Mol. Cancer Ther. 3: 223-232

15. Jin $\mathrm{G}$ and Howe PH (1999) Transforming growth factor beta regulates clusterin gene expression via modulation of transcription factor c-Fos. Eur. J. Biochem. 263: 534-542

16. Yang CR, Yeh S, Leskov K, Odegaard E, Hsu HL, Chang C, Kinsella TJ, Chen DJ and Boothman DA (1999) Isolation of Ku70-binding proteins (KUBs). Nucleic Acids Res. 27: 2165-2174

17. Criswell T, Klokov D, Beman M, Lavik JP and Boothman DA (2003) Repression of IR-inducible clusterin expression by the p53 tumour suppressor protein. Cancer Biol. Ther. 2: 372-380

18. Zellweger T, Kiyama S, Chi K, Miyake H, Adomat H, Skov K and Gleave ME (2003) Overexpression of the cytoprotective protein clusterin decreases radiosensitivity in the human LNCaP prostate tumour model. BJU Int. 92: 463469

19. Miyake H, Hara I, Kamidono S, Gleave ME and Eto H (2003) Resistance to cytotoxic chemotherapy induced apoptosis in human prostate cancer cells is associated with intracellular clusterin expression. Oncol. Rep. 10: 469-4673

20. Osborne JE and Hutchinson PE (2002) Vitamin D and systemic cancer: is this relevant to malignant melanoma? Br. J. Dermatol. 147: 197-213

21. Cervellera M, Raschella G, Santilli G, Tanno B, Ventura A, Mancini C, Sevignani C, Calabretta B and Sala A (2000) Direct transactivation of the antiapoptotic gene apolipoprotein J (clusterin) by B-MYB. J. Biol. Chem. 275: 21055-21060

22. Sankilli G, Aronow BJ and Sala A (2003) Essential requirements of apolipoprotein $\mathrm{J}$ (clusterin) signalling for $\mid \kappa \mathrm{B}$ expression and regulation of NF- $\kappa$ B activity. J. Biol. Chem. 278: 38214-38219

23. Bettuzzi S, Astancolle S, Guidentti G, Moretti M, Tiozzo R and Corti A (1999) Clusterin (SGP-2) gene expression is cell cycle dependent in normal human dermal fibroblasts. FEBS Lett. 448: 297-300

24. Yang CR, Leskov K, Hosley-Eberlein K, Criswell T, Pink J, Kinsella T and Boothman DA (2000) Nuclear clusterin/XIP8, an X-ray-induced Ku70-binding protein that signals cell death. Nucleic Acids Res. 97: 5907-5912 
25. Leskov K, Criswell T, Antonio S, Li J, Yang CR, Kinsella TJ and Boothman DA (2001) When X-ray-inducible proteins meet DNA double strand break repair Semin. Radiat. Oncol. 11: 352-372

26. Sawada M, Hayes P and Matsuyama S (2003) Cytoprotective membranepermeable peptides designed from the Bax-binding domain of Ku70. Nat. Cell Biol. 5: 352-357

27. Matuda A, Itoh Y, Koshikawa N, Akizawa T, Yana I and Sieki M (2003) Clusterin: an abundant serum factor, is a possible negative regulator of MT6 MMP/MMP-25 produced by neutrophils. J. Biol. Chem. 278: 36350-36357

28. Seiberg M and Marthinuss J (1995) Clusterin expression within skin correlates with hair growth. Dev. Dyn. 202: 294-301

29. Thomas-Salgar S and Millis AJ (1994) Clusterin expression in differentiating smooth muscle cells. J. Biol. Chem. 269: 17879-17885

30. Simboli-Campbell M, Narvaez CJ, van Weelden K, Tenniswood M and Welsh J (1997) Comparative effects of $1,25(\mathrm{OH})_{2} \mathrm{D}_{3}$ and EB1089 on cell cycle kinetics and apoptosis in MCF-7 breast cancer cells. Breast Cancer Res. Treat. 42: $31-$ 41

31. James SY, Mackay AG and Colston KW (1996) Effects of $1,25(\mathrm{OH})_{2} \mathrm{D}_{3}$ and its analogues on induction of apoptosis in breast cancer cells. J. Steroid Biochem. Mol. Biol. 58: 395-401

32. Narvaez CJ, Vanweelden K, Byrne I and Welsh J (1996) Characterization of vitamin D3-resistant MCF-7 cell line. Endocrinology 137: 400-409

33. Crescioli C, Ferruzzi P, Caporali A, Scaltriti M, Bettuzzi S, Mancina R, Gelmini S, Serio M, Villari D, Vannelli GB, Colli E, Adorini L and Maggi M (2004) Inhibition of prostate cell growth by BXL-628, a calcitriol analogue selected for a phase II clinical trial in patients with benign prostate hyperplasia. Eur. J. Endocrinol. 150: 591-603

34. Gutacker C, Klock G, Diel P and Kock-Brabdt C (1999) Nerve growth factor and epidermal growth factor stimulate clusterin gene expression in PC12 cells. Biochem. J. 339: 759-766

35. Wegrowski Y, Perreau C, Martiny L, Haye B, Maquart FX and Bellon C (1999) Transforming growth factor beta-1 up-regulates clusterin synthesis in thyroid epithelial cells. Exp. Cell Res. 247: 475-483

36. Araki S, Leskov K, Klokov D, Kang S-W, Stavnezer E, Danielpour D and Boothman DA (2005) Regulation of the clusterin promoter by TGF- $\beta 1$ involves Smad3 activation and binding. Mol. Cell. Biol. (submitted)

37. Scaltriti M, Brausi M, Amorosi A, Caporali A, D'Arca D, Astancolle S, Corti A and Bettuzzi S (2004) Clusterin (SGP-2, APOJ) expression is downregulated in low- and high-grade human prostate cancer. Int. J. Cancer 108: 23-30

38. Redondo M, Villar E, Torres-Munoz J, Tellez T, Morell M and Petito CK (2002) Overexpression of clusterin in human breast carcinoma. Am. J. Pathol. 157: 393-399

39. Chen X, Halberg RB, Ehrhardt WM, Torrealba J and Dover WF (2003) Clusterin as a biomarker in murine and human intestinal neoplasia. Proc. Natl. Acad. Sci. USA 100: 9530-9535
40. Thomas-Tikhonenko A, Viard-Leveugle I, Dews M, Wehrli P, Sevignani $C$, Yu D, Ricci S, El-Deiry W, Aronow B, Kaya G, Saurat JH and French LE (2004) Myc-transformed epithelial cells down-regulate clusterin, which inhibits either growth in vitro and carcinogenesis in vivo. Cancer Res. 61: 3126-3136

41. Zhang LY, Ying WT, Mao YS, He HZ, Liu Y, Wang HX, Liu F, Wang K and Zha $\mathrm{XH}$ (2003) Loss of clusterin both in serum and tissue correlates with the tumorigenesis of oesophageal squamous cell carcinoma via proteomic approaches. World J Gastroenterol 9: 650-654

42. Xie MJ, Motoo Y, Su SB, Mouri H, Ohtsubo K, Matsubara F and Sawabu N (2002) Expression of clusterin in human pancreatic cancer. Pancreas 25: 234238

43. Saffer H, Wahed A, Rassidakis GZ and Medeiros LJ (2002) Clusterin expression in malignant lymphomas. Mod. Pathol. 15: 1221-1223

44. July LV, Akbari M, Zellweger T, Jones EC, Goldenberry SL and Gleave ME (2002) Clusterin expression is significantly enhanced in prostate cancer cells following androgen withdrawal therapy. Prostate 50: 179-188

45. Biroccio A, D'angel C, Jansen B, Gleave ME and Zupi G (2005) Antisense clusterin oligonucleotides increase the response of HER-2 gene amplified breast cancer cells to Trastuzumab. J. Cell. Physiol. 204: 463-469

46. Gleave ME and Monia BP (2005) Antisense therapy for cancer. Nat. Rev. Cancer. 5: 468-479

47. Yamanaka K, Gleave ME, Hara I, Muramaki M and Miyake H (2005) Synergistic antitumor effect of combined use of adenoviral-mediated p53 gene transfer and antisense oligodeoxynucleotide targeting clusterin gene in an androgen-independent human prostate cancer model. Mol. Cancer Ther. 4: 187-195

48. Zellweger T, Miyake H, July LV, Akbari M, Kiyama S and Gleave ME (2001) Chemosensitization of human renal cell cancer using antisense oligonucleotides targeting the antiapoptotic gene clusterin. Neoplasia 3: 360367

49. Gleave M and Jansen B (2003) Clusterin and IGFBPs as antisense targets in prostate cancer. Ann. NY Acad. Sci. 1002: 95-104

50. Criswell T, Beman M, Araki S, Leskov K, Cataldo E, Mayp LD and Boothman DA (2005) Delayed activation of insulin-like growth factor-1 receptor/Src/ MAPK/Egr-1 signaling regulates clusterin expression, a pro-survival factor. J. Biol. Chem. 280: 14212-14221

51. Zellweger T, Miyake H, Cooper S, Chi K, Conklin BS, Monia BP and Gleave ME (2001) Antitumor activity of antisense clusterin oligonucleotides is improved in vitro and in vivo by incorporation of 2'-0-(2-methoxy) ethyl chemistry. J. Pharmacol. Exp. Ther. 298: 934-940

52. Chung J, Kwak C, Jin RJ, Lee CH, Lee KH and Lee SE (2004) Enhanced chemosensitivity of baldder cancer cells to cisplatin by suppression of clusterin in vitro. Cancer Lett. 203: 155-161 